GESCHICHTE

DER

PHILOSOPHIE 


\section{CHRISTOPH HELFERICH}

\section{GESCHICHTE \\ DER \\ PHILOSOPHIE}

Von den Anfängen

bis zur Gegenwart

und

Östliches Denken

Dritte Auflage

mit 192 Abbildungen

mit einem Beitrag von

Peter Christian Lang

VERLAG J. B. METZLER

STUTTGART · WEIMAR 
Umschlagabbildung siehe Bildlegende auf S. 5

Die Deutsche Bibliothek - CIP-Einheitsaufnahme

Geschichte der Philosophie: von den Anfängen bis zur Gegenwart und östliches Denken / Christoph Helferich.

Mit einem Beitr. von Peter Christian Lang.

3. Aufl. - Stuttgart; Weimar: Metzler, 2001

1. Aufl. u. d. T.: Helferich, Christoph: Geschichte der Philosophie ISBN 978-3-476-01522-8

NE: Helferich, Christoph; Lang, Peter Christian

ISBN 978-3-476-01522-8

ISBN 978-3-476-03175-4 (eBook)

DOI 10.1007/978-3-476-03175-4

Dieses Werk einschließlich aller seiner Teile ist urheberrechtlich geschützt. Jede Verwertung außerhalb der engen Grenzen des Urheberrechtsgesetzes ist ohne Zustimmung des Verlages unzulässig und strafbar. Das gilt insbesondere für Vervielfältigungen, Übersetzungen, Mikroverfilmungen und die Einspeicherung und Verarbeitung in elektronischen Systemen.

(C) 2001 Springer-Verlag GmbH Deutschland

Ursprünglich erschienen bei J. B. Metzlersche Verlagsbuchhandlung und Carl Ernst Poeschel Verlag GmbH in Stuttgart 2001 www.metzlerverlag.de info@metzlerverlag.de 


\section{INHALT}

Vorwort $\ldots \ldots \ldots \ldots \ldots \ldots \ldots$ VII

\section{Die Philosophie der Antike}

Die Anfänge der griechischen Philosophie . Der Mensch als neues Problem:

Sokrates und die Sophisten . . . . . . . . 13

Die Ausprägung der klassischen

griechischen Philosophie .......... 24

Die philosophischen Schulen im Zeitalter

des Hellenismus . . . . . . . . . . . . . . . 54

Der Neuplatonismus, die Philosophie

der heidnischen Spätantike . . . . . . . . . . 63

\section{Die Philosophie}

\section{des christlichen Mittelalters}

Philosophie und Theologie

in der Spätantike . . . . . . . . . . . . . . 70

Die Spannung zwischen Glaube

und Vernunft in der Scholastik

\section{Humanismus, Reformation und die Umwälzung des Wissens von der Natur}

"Rináscita«, Wiedergeburt der Antike im italienischen Humanismus . . . . . . . . . 117

Reformation der Kirche oder Reich Gottes auf Erden oder »Geist des Kapitalismus«? . . 122 Träumende Vernunft, männliche Vernunft, privateVernunft . . . . . . . . . . 128 Die Erde ist gar nicht Mittelpunkt der Welt! - Über die Umwälzung von Weltbildern durch wissenschaftliche Revolutionen . . . . 136 Die faustische Renaissance . . . . . . . . 147 »Knowledge is power«: Francis Bacon und die Royal Society of London for Improving Natural Knowledge . .

\section{Die Philosophen der neuen,} der bürgerlichen Zeit

Neuzeit - was heißt das?

Die großen Systeme der konstruierenden

Vernunft und ihre Kritiker Pascal und Vico . 160

1 Der englische Empirismus-Philosophie der Erfahrung und des "gesunden «,

d.h. bürgerlichen Menschenverstandes . . 187

Die Aufklärung-eine gesamteuropäische

Bewegung . . . . . . . . . . . . . . . . 203

Die Abenteuer der Vernunft

im deutschen Idealismus . . . . . . . . . . . 245

Das neuzeitliche Jahrhundert:

\section{Philosophie}

in der Maschinenwelt

Fortschritt-Stichwort zur Zeit . . . . . . . 293

Der »alte Positivismus « in Frankreich,

England und Deutschland . . . . . . . . . . 295

Die kritisch träumende Vernuft

der (utopischen) (Früh-)Sozialisten . . . . . . 303

Vormärz-Es gärt in Deutschland:

Die Hegelschule spaltet sich:

Ludwig Feuerbach will

den »ganzen Menschen« . . . . . . . . . . . 308

Begreifen der Praxis - Karl Marx

und Friedrich Engels . . . . . . . . . . . . . . . . 314

Einspruch der Anarchisten . . . . . . . . . 335

Drei Einzelgänger . . . . . . . . . . . 338

Exakte Naturwissenschaften, geschicht-

liche Welt, der handelnde Mensch -

Themen der Universitätsphilosophie

um die Jahrhundertwende . . . . . . . . 358

\section{Die Philosophie unserer Zeit}

Albert Einstein, Sigmund Freud, Wassily Kandinsky - drei Namen für die Erweiterung und Verrätselung der Wirklichkeit im 20. Jahrhundert . . . . . 373 
»Linguistic turn «, d.h. die Wendung zur Sprache in der modernen Philosophie englischer Prägung . .

Formen des Marxismus . . . . . . . . . . . . . 389

Existenz als Thema . . . . . . . . . . . . . . . 399

Erkenntnis wird »Erkenntnis«,

»völkische Weltanschauung « oder

»Seelenmystik « im Faschismus . . . . . . . . . 409

Stichwörter zur Gegenwart . . . . . . . . . 416

Lebendige Philosophie:

Debatten und Kontroversen der siebziger und achtziger Jahre (von Peter Christian Lang)

Eine neue Generation tritt auf 447 Positionen der praktischen Philosophie und Ethik

Positionen der Ästhetik 464

\section{Wegweiser in die Philosophie} des Ostens

Ost und West . . . . . . . . . . . . . . 484

Indien: Das Abenteuer der Suche

nach dem Selbst . . . . . . . . . . 487

Nirvāna-»Verwehen« .............. 496

Yin und Yang, "einander entgegengesetzt,

einander ergänzend «. Die Ordnung

der Welt im chinesischen Denken . . . . . . 512

In den Gärten des Zen . . . . . . . . . . . 534

\section{Anhang}

Anmerkungen . . . . . . . . . . . 543

Einführende Bibliographie . . . . . . . . . 555

Personen- und Werkregister . . . . . . . . 557

Sachregister . . . . . . . . . . . . . . . 565

Bildquellen . . . . . . . . . . . . . 571 


\section{VORWORT}

Auf den ersten Blick mag die Geschichte der Philosophie wie ein Labyrinth erscheinen, so viele Gestalten hat sie in ihrem Verlauf hervorgebracht und so fremdartig mag die Sprache wirken, die diese sprechen. Man sollte sich davon jedoch keinesfalls beirren lassen. Denn sehr schnell wird man die Erfahrung machen, daß diese scheinbar verwirrende Vielfalt in Wirklichkeit ein großes Angebot ist. Das Angebot, teilzunehmen an dem spannenden Versuch, Antworten zu finden auf Lebensfragen, wie sie anderen Zeiten sich gestellt haben und wie unsere Zeit sie stellt. Die Geschichte der Philosophie ist die gespeicherte Erfahrung dieser Versuche. Und man wird sehr schnell spüren, daß man in der Auseinandersetzung mit ihnen nicht derselbe bleibt. Denn gerade der Abstand zur Gegenwart, den man dadurch gewinnt, bietet die Chance, sich selbst und die eigene Zeit in einem neuen Licht zu sehen. Indem so viele Denk- und Verhaltensgewohnheiten, das scheinbar Normalste plötzlich fragwürdig werden, eröffnet die Geschichte der Philosophie einen neuen Raum. Sie schafft Luft, kritische Distanz, neben dem Willen dazu die vielleicht wichtigste Voraussetzung von Freiheit.

In ihrem Aufbau, der Epochengliederung und der Auswahl der repräsentativen Namen folgt die vorliegende Darstellung einer überlieferten Form von Philosophiegeschichtsschreibung. Mit voller Absicht, denn sie ist für einen breiten Kreis von Lesern gedacht, die einen Zugang zur Philosophie gewinnen wollen über ihre geschichtliche Gestalt. Wohl aber habe ich versucht, in der Darbietung selbst das Bewußtsein wachzuhalten, daß diese überlieferte Form, ihr Bestand und die Art seiner Gliederung nichts Selbstverständliches ist. Die Frage z. B., ob das östliche Denken überhaupt in den Zusammenhang einer Geschichte der Philosophie hineingehört, zeigt das nur besonders deutlich. Es ist außerdem klar, daß sowohl bei den Epochen als auch den einzelnen Philosophen selbst immer eine Auswahl getroffen werden mußte. Mein Ziel war dabei, die Schwerpunkte so zu setzen, daß jeweils ein möglichst breites und interessantes Spektrum von philosophischen und geschichtlichen Fragestellungen, Problemen und Perspektiven zur Sprache kommt, von denen jeder einzelne Aspekt im Idealfall geeignet ist, zum Nachdenken über bestimmte grundlegende Sachverhalte anzuregen. Denn meist hat die Philosophie ihre Fragen beim ersten Auftauchen bereits so klar und zugespitzt gestellt, daß die Auseinandersetzung mit diesen klassischen Formulierungen für den Anfänger den größten Erkenntnisgewinn verspricht. Das ist der entscheidende Vorteil der Geschichte der Philosophie. Und wenn einige ihrer Probleme und Denkformen heute nicht mehr oder so nicht mehr da sind oder dazusein scheinen umso mehr Anlaß, stutzig zu werden, umso mehr Anlaß zur Verwunderung. Womit, nach einem alten Wort des Aristoteles, die Philosophie beginnt.

Es liegt in der Natur der Sache, daß alle bedeutenden Philosophen große Meister der Sprache und oft sogar Sprachschöpfer gewesen sind. Gegenüber der gefährlichen Sorglosigkeit, die in unserer Zeit im Umgang mit der Sprache herrscht, ist diese Aufmerksamkeit der Philosophie auf die Sprache vielleicht wichtiger als je zuvor. Dabei hat sie auch eine eigene Sprache 
entwickelt, in der sie ihre Probleme behandelt. Hält man sich die Entwicklung anderer Wissenschaften vor Augen, so ist das eigentlich selbstverständlich. Aber gerade diese besondere philosophische Terminologie ist es, die von dem Laien häufig als Barriere empfunden wird. Ich habe versucht, beiden Ansprüchen zu genügen, d.h. die Anzahl der Fachwörter begrenzt zu halten und diese so zu erklären bzw. zu übersetzen, daß sie verständlich sind. Andererseits aber die Fachwörter, wo es sinnvoll und notwendig ist, auch wirklich einzubringen, denn die Bekanntschaft mit ihnen, das Sachwissen, gehört zur Sache hinzu. Darüber hinaus sind die Schriften der Philosophen reich an manchmal komischen und amüsanten, oft aber auch wunderbar dichten und bildkräftigen Formulierungen. Diese Seite der Philosophie, der Umgang mit ihren gelungenen sprachlichen Bildern, hat mir bei der Arbeit an diesem Buch besondere Freude bereitet; ich wünsche mir, daß sie sich auf die Leser überträgt.

Bleibt noch die angenehme Aufgabe, allen zu danken, die mir geholfen haben, indem sie Teile gelesen und mit mir besprochen haben. Mein besonderer Dank gilt dem J. B. Metzler Verlag und seinem Lektor Bernd Lutz, mit dessen »altem Plan « alles anfing. Soviel Dank Nicola, daß sie mich so oft so nachsichtig »an den Büchern « gelassen hat und auch nicht, zum Glück.

Florenz, im März 1985

Christoph Helferich

\section{Vorwort zur zweiten Auflage}

Die Zustimmung, die das Buch in der Kritik der Fachwelt erhielt, und die Aufnahme, die es in einer breiten Leserschaft bis in die Praxis des Philosophieunterrichts selbst hinein erfuhr, haben mir große Freude bereitet. Sie bestätigen, daß Auswahlprinzip, Themenfassung und Darstellungsweise insgesamt als gelungen betrachtet werden dürfen. Daß dabei manchem Kritiker dieser oder jener Autor bzw. die von ihm vertretene Richtung als zu kurz gekommen scheint, kann in diesem Zusammenhang eigentlich nicht verwundern. Es verweist aber auf das Risiko, dem.das Unternehmen einer Geschichte der Philosophie nach wie vor ausgesetzt bleibt.

Die vorliegende zweite Auflage wurde um einige Beiträge zur italienischen Philosophiegeschichte erweitert, die für eine italienische Ausgabe des Werkes geschrieben wurden; die Hineinnahme dieser interessanten Linie vermag nicht zuletzt den gesamteuropäischen Charakter der philosophiegeschichtlichen Tradition zu unterstreichen. Weitere Veränderungen und Ergänzungen betreffen vor allem die Philosophie unseres Jahrhunderts, wobei ja noch gar nicht abzusehen ist, welche tiefgreifenden Wandlungen die allgemeine Entwicklung - Stichwort 9. November ' 89 auch für das Denken nach sich ziehen wird. Die Überarbeitung dieses Kapitels wurde von Peter Christian Lang vorgenommen, der auch, ausgehend von der Entwicklung in Deutschland, in einem eigens für diese Ausgabe verfaßten Beitrag die Diskussion der Gegenwartsphilosophie weiterführt, was auch dieser zweiten Auflage unverminderte Aktualität sichert. Sie erscheint in einem neuen graphischen Gewand, welches die Lesbarkeit erleichtert; darüber hinaus wurden das Bildmaterial vermehrt und eine einführende Bibliographie erstellt, die dem Leser eine rasche Orientierung erlaubt.

Florenz, im April 1991

Christoph Helferich 